\title{
Kaon versus Antikaon Production at SIS Energies*
}

\author{
W. Cassing, E. L. Bratkovskaya, U. Mosel, S. Teis and A. Sibirtsev \\ Institut für Theoretische Physik, Universität Giessen \\ 35392 Giessen, Germany
}

\begin{abstract}
We analyse the production and propagation of kaons and antikaons in $\mathrm{Ni}+\mathrm{Ni}$ reactions from $0.8-1.85 \mathrm{GeV} / \mathrm{u}$ within a coupled channel transport approach including the channels $B B \rightarrow K^{+} Y N, \pi B \rightarrow K^{+} Y, B B \rightarrow N N K \bar{K}, \pi B \rightarrow$ $N K \bar{K}, K^{+} B \rightarrow K^{+} B, \bar{K} B \rightarrow \bar{K} B, Y N \rightarrow \bar{K} N N, \pi \pi \rightarrow K \bar{K}$ as well as $\pi Y \rightarrow$ $\bar{K} N$ and $\bar{K} N \rightarrow \pi Y$ for the antikaon absorption. Whereas the experimental $K^{+}$ spectra can be reproduced without introducing any selfenergies for the mesons in $\mathrm{Ni}+\mathrm{Ni}$ collisions from 0.8 to $1.8 \mathrm{GeV} / \mathrm{u}$, the $K^{-}$yield is underestimated by a factor of $5-7$ at 1.66 and $1.85 \mathrm{GeV} / \mathrm{u}$. However, introducing density dependent antikaon masses as proposed by Kaplan and Nelson, the antikaon spectra can be reasonably well described.
\end{abstract}

*supported by GSI Darmstadt 


\section{Introduction}

The study of hot and dense nuclear matter via relativistic nucleus-nucleus collisions is the major aim of high energy heavy-ion physics. Nowadays, the search for a restoration of chiral symmetry at high baryon density or for a phase transition to the quark-gluon plasma (QGP) is of specific interest. However, any conclusions about the hadron properties at high temperature or baryon densities must rely on the comparison of experimental data with theoretical approaches based on nonequilibrium kinetic theory. Among these, the covariant RBUU approach [1, 2, 3, 4, 5, 6, 7, 8, 9], the QMD [10] or RQMD model [11, and more recently the HSD approach [12 have been successfully used in the past. As a genuine feature of transport theories there are two essential ingredients: i.e. the baryon (and meson) scalar and vector selfenergies - which are neglected in many approaches - as well as in-medium elastic and inelastic cross sections for all hadrons involved.

Selfenergy effects in the production of particles have been found previously for antiprotons by a number of groups [3, 6, 13, 14] though the actual magnitude of the attractive $\bar{p}$-potential in the nuclear medium is still a matter of debate. The first dynamical studies on antikaon production in nucleus-nucleus collisions have been performed since more than a decade ago without including any selfenergies for the mesons

produced [15, 16, 17. However, as advocated in 118, 19, 20, 21, 22, 23, 24, 25 also antikaons should feel strong attractive forces in the medium so that their production threshold should be reduced at finite baryon density. A first exploratory study with respect to antikaon selfenergies has been performed by Li, Ko and Fang in Ref. [26] with the result that sizeable $K^{-}$potentials are needed to explain the experimental spectra from [27] for $\mathrm{Ni}+\mathrm{Ni}$ at $1.85 \mathrm{GeV} / \mathrm{u}$. Similar, but less pronounced results have been obtained in Ref. [12] at AGS energies where especially the kaon/pion ratio is much better reproduced when including kaon selfenergies. However, at $10-15 \mathrm{GeV} / \mathrm{u}$ there is also a sizeable kaon production from meson-meson channels with partly unknown cross sections, which contribute to the $K^{+} K^{-}$yield. Thus heavy-ion collisions in the range of only 1-2 GeV/u are more favourable systems for the study of kaon dynamics since the number of production channels is much more restricted while already considerable baryon densities (up to $3 \times \rho_{0} \approx 0.5 \mathrm{fm}^{-3}$ ) can be reached in the compression phase [1].

The most serious problem related to $K^{-}$production at 'subthreshold' energies are the baryon-baryon and pion-baryon elementary production cross sections close to threshold where no experimental data are available so far. It has recently been argued that extrapolations from high energy data [28] used in [26] overestimate the elementary $K^{-}$yield by more than an order of magnitude close to threshold [29, 30, 31] such that a reanalysis of this question in nucleus-nucleus reactions appears necessary. In previous works we have calculated - within boson exchange models - the near threshold production cross section for antiprotons [32], vector mesons $(\rho, \omega, \Phi)$ [33] as well as antikaons [31] from nucleon-nucleon as well as pion-nucleon reactions (cf. also Ref. [34] in case of $K^{+}$mesons). Since within the boson exchange model one can interpolate between different isospin channels and thus compare to a much larger set of experimental data, the results of these studies should be more reliable than the early parametriza- 
tions [28, 35, 36], that partly are in severe contradiction to the available phase space.

Our work thus is organized as follows: we start in Sect. 2 with a brief description of the transport approach employed and specify the kaon selfenergies incorporated in the calculation as well as the various kaon production and reabsorption channels that are taken into account. Section 3 is devoted to a presentation of our calculated results for $\mathrm{Ni}+\mathrm{Ni}$ at $0.8-1.85 \mathrm{GeV} / \mathrm{u}$ in comparison to published experimental data. We will include also a differential analysis of the various production channels contributing to the antikaon production and explore the effect of kaon selfenergies. Section 4, finally, is devoted to a summary and discussion of open problems.

\section{Ingredients of the transport approach}

In this paper we perform our analysis along the line of the HSD" approach [12] which is based on a coupled set of covariant transport equations for the phase-space distributions $f_{h}(x, p)$ of hadron $h[7$, 12], i.e.

$$
\begin{aligned}
& \left\{\left(\Pi_{\mu}-\Pi_{\nu} \partial_{\mu}^{p} U_{h}^{\nu}-M_{h}^{*} \partial_{\mu}^{p} U_{h}^{S}\right) \partial_{x}^{\mu}+\left(\Pi_{\nu} \partial_{\mu}^{x} U_{h}^{\nu}+M_{h}^{*} \partial_{\mu}^{x} U_{h}^{S}\right) \partial_{p}^{\mu}\right\} f_{h}(x, p) \\
& \quad=\sum_{h_{2} h_{3} h_{4} \ldots} \int d 2 d 3 d 4 \ldots\left[G^{\dagger} G\right]_{12 \rightarrow 34 \ldots} \delta^{4}\left(\Pi+\Pi_{2}-\Pi_{3}-\Pi_{4} \ldots\right) \\
& \quad \times\left\{f_{h_{3}}\left(x, p_{3}\right) f_{h_{4}}\left(x, p_{4}\right) \bar{f}_{h}(x, p) \bar{f}_{h_{2}}\left(x, p_{2}\right)\right. \\
& \left.\quad-f_{h}(x, p) f_{h_{2}}\left(x, p_{2}\right) \bar{f}_{h_{3}}\left(x, p_{3}\right) \bar{f}_{h_{4}}\left(x, p_{4}\right)\right\} \ldots .
\end{aligned}
$$

In Eq. (1) $U_{h}^{S}(x, p)$ and $U_{h}^{\mu}(x, p)$ denote the real part of the scalar and vector hadron selfenergies, respectively, while $\left[G^{+} G\right]_{12 \rightarrow 34 \ldots} \delta^{4}\left(\Pi+\Pi_{2}-\Pi_{3}-\Pi_{4} \ldots\right)$ is the 'transition rate' for the process $1+2 \rightarrow 3+4+\ldots$ which is taken to be on-shell in the semiclassical limit adopted. The hadron quasi-particle properties in (1) are defined via the mass-shell constraint [7],

$$
\delta\left(\Pi_{\mu} \Pi^{\mu}-M_{h}^{* 2}\right)
$$

with effective masses and momenta (in local Thomas-Fermi approximation) given by

$$
\begin{aligned}
M_{h}^{*}(x, p) & =M_{h}+U_{h}^{S}(x, p) \\
\Pi^{\mu}(x, p) & =p^{\mu}-U_{h}^{\mu}(x, p)
\end{aligned}
$$

while the phase-space factors

$$
\bar{f}_{h}(x, p)=1 \pm f_{h}(x, p)
$$

are responsible for fermion Pauli-blocking or Bose enhancement, respectively, depending on the type of hadron in the final/initial channel. The dots in Eq. (11) stand for further contributions to the collision term with more than two hadrons in the final/initial channels. The transport approach (11) is fully specified by $U_{h}^{S}(x, p)$ and

\footnotetext{
${ }^{1}$ Hadron String Dynamics
} 
$U_{h}^{\mu}(x, p)(\mu=0,1,2,3)$, which determine the mean-field propagation of the hadrons, and by the transition rates $G^{\dagger} G \delta^{4}(\ldots)$ in the collision term, that describes the scattering and hadron production/absorption rates.

The scalar and vector mean fields $U_{h}^{S}$ and $U_{h}^{\mu}$ for baryons are taken from Ref. [12] and don't have to be specified here again. In the present approach we propagate explicitly pions, kaons, and $\eta$ 's and assume that the pions as Goldstone bosons do not change their properties in the medium; we also discard selfenergies for the $\eta$-mesons in the present calculation which have a minor effect on the kaon dynamics. The kaonbaryon interactions are described along the line of Kaplan and Nelson [20] and have to be specified more explicitly.

\subsection{Kaon selfenergies}

As in case of antiprotons there are a couple of models for the kaon and antikaon selfenergies [18, 19, 20, 21, 22, 23, 24, 25], which differ in the actual magnitude of the selfenergies, however, agree on the relative signs for kaons and antikaons. Thus in line with the kaon-nucleon scattering amplitude the $K^{+}$potential should be slightly repulsive at finite baryon density whereas the antikaon should see an attractive potential in the nuclear medium. Without going into a detailed discussion of the various models we adopt the more practical point of view, that the actual $K^{+}$and $K^{-}$selfenergies are unknown and as a guide for our analysis adopt the approach by Kaplan and Nelson 20.

Starting from a $S U(3)_{L} \times S U(3)_{R}$ chiral Lagrangian and using chiral perturbation theory Kaplan and Nelson [20] have derived an effective meson-baryon Lagrangian up to first order in the baryon scalar density, which they claim to be valid up to $\sim 7 \rho_{0}$. Since the coefficients in this Lagrangian are approximately known experimentally (within an uncertainty of about 30\%), one can model a Lagrangian of lower complexity, but with the same properties on the mean-field level. Such limits lead to the following dispersion relation for kaons in the nuclear medium [37, 38]:

$$
\begin{aligned}
& \omega_{K^{+}}(x, \mathbf{p})=\left\{\mathbf{p}^{2}+m_{K}^{2}\left(1-\frac{\Sigma_{K N}}{f_{K}^{2} m_{K}^{2}} \rho_{S}(x)+\left(\frac{3 \rho_{B}(x)}{8 f_{K}^{2} m_{K}}\right)^{2}\right)\right\}^{1 / 2}+\frac{3}{8} \frac{\rho_{B}(x)}{f_{K}^{2}}, \\
& \omega_{K^{-}}(x, \mathbf{p})=\left\{\mathbf{p}^{2}+m_{K}^{2}\left(1-\frac{\Sigma_{K N}}{f_{K}^{2} m_{K}^{2}} \rho_{S}(x)+\left(\frac{3 \rho_{B}(x)}{8 f_{K}^{2} m_{K}}\right)^{2}\right)\right\}^{1 / 2}-\frac{3}{8} \frac{\rho_{B}(x)}{f_{K}^{2}},
\end{aligned}
$$

with $m_{K}$ denoting the bare kaon mass, $f_{K} \approx 93 \mathrm{MeV}$ and $\Sigma_{K N} \approx 350 \mathrm{MeV}$, while $\rho_{S}(x)$ and $\rho_{B}(x)$ are the scalar and vector baryon densities at space-time position $x$, respectively. We note, that the kaon quasi-particle dynamics in the transport approach (1) are completely determined by the space and momentum derivatives of (5) according to the Hamilton equations.

In order to evaluate the dispersion relations (5) we need a model to compute the scalar density $\rho_{S}$ as a function of the baryon density $\rho_{B}$. Here, the relativistic RBUU model [7] provides a useful guide. Within a good approximation the nucleon effective 
mass for nuclear matter at temperature $\mathrm{T}=0$ can be written as

$$
m_{N}^{*}=\frac{m_{N}}{1+0.52 \frac{\rho_{B}}{\rho_{0}}},
$$

where $m_{N}$ is the vacuum nucleon mass and $\rho_{0} \approx 0.16 \mathrm{fm}^{-3}$. The scalar density then can be evaluated by

$$
\rho_{S}\left(p_{F}\right)=\frac{g}{2 \pi^{2}} \int_{0}^{p_{f}} d p p^{2} \frac{m^{*}}{\sqrt{p^{2}+m^{* 2}}}
$$

with the Fermi momentum

$$
p_{F}=\left(\frac{3}{2 \pi^{2}} \rho_{B}\right)^{1 / 3}
$$

and $g=4$ for isospin symmetric matter.

In the restframe of the kaons the $K^{+}$and $K^{-}$energy/mass changes almost linearly with the baryon density as shown in Fig. 1. Since the $K^{+}$mass changes only very modestly with the baryon density, we will discard any selfenergy effects on the $K^{+}$mesons in the present analysis; this approximation will be confronted with the experimental data in Sect. 3.

For practical purposes, furthermore, we include the $K^{-}$selfenergy in a density dependent quasi-particle mass as

$$
m_{K}^{*}\left(\rho_{B}\right)=m_{K}^{0}\left(1-\alpha \frac{\rho_{B}}{\rho_{0}}\right)
$$

with $\alpha \approx 0.2$ (cf. lower dashed line in Fig. 四 ${ }^{2}$. Linear fits to the antikaon selfenergies from [25, 24] lead to different values for the parameter $\alpha$ in the range $0.1 \leq \alpha \leq 0.4$. We note that the dropping of the antikaon mass is associated with a corresponding scalar energy density in the baryon/meson Lagrangian, such that the total energy-momentum is conserved during the heavy-ion collision (cf. [12]).

\subsection{Kaon and antikaon reaction channels}

First, the individual production channels of the kaons have to be specified. Here, we prefer to express the cross sections as a function of the scaled invariant energy squared $s_{0} / s$, since the change of the quasi-particle mass then can be incorporated in the threshold energy $\sqrt{s_{0}}$ for the particular channel. This recipe might be still a matter of debate; however, our findings in Refs. [29, 30, 31, 32, 33] indicate, that the production is essentially dominated by phase space close to threshold and thus a scaling in $s_{0} / s$ should be a good approximation.

The isospin averaged production cross section of a $K^{+} \Lambda$ and $K^{+} \Sigma$ pair in a nucleonnucleon collision is related to the measured isospin channels as:

$$
\sigma_{N N \rightarrow K^{+} \Lambda N}=\frac{3}{2} \sigma_{p p \rightarrow K^{+} \Lambda p}
$$

\footnotetext{
${ }^{2}$ In Ref. 12] we have used $\alpha=0.16$ in order to get a linear fit up to $6 \times \rho_{0}$ as needed for the AGS energy regime.
} 


$$
\sigma_{N N \rightarrow K^{+} \Sigma N}=\frac{3}{2}\left(\sigma_{p p \rightarrow K^{0} \Sigma^{+} p}+\sigma_{p p \rightarrow K^{+} \Sigma^{0} p}\right)
$$

Following [30] the reaction cross section can be approximated by

$$
\begin{aligned}
& \sigma_{p p \rightarrow K^{+} \Lambda p}(s)=732\left(1-\frac{s_{01}}{s}\right)^{1.8}\left(\frac{s_{01}}{s}\right)^{1.5}[\mu b] \\
& \sigma_{p p \rightarrow K^{0} \Sigma^{+} p}(s)=338.46\left(1-\frac{s_{02}}{s}\right)^{2.25}\left(\frac{s_{02}}{s}\right)^{1.35}[\mu b] \\
& \sigma_{p p \rightarrow K^{+} \Sigma^{0} p}(s)=275.27\left(1-\frac{s_{02}}{s}\right)^{1.98}\left(\frac{s_{02}}{s}\right)[\mu b]
\end{aligned}
$$

with $\sqrt{s_{01}}=m_{\Lambda}-m_{N}+m_{K}^{0}$ and $\sqrt{s_{02}}=m_{\Sigma}+m_{N}+m_{K}^{0}$. According to isospin relations the $N \Delta$ and $\Delta \Delta$ production channels get additional factors of $3 / 4$ and $1 / 2$, respectively. The elementary cross sections for the pion induced channels $\pi N \rightarrow K^{+} Y$ have been computed by Tsushima et al. in Ref. [39], which we adopt for our present study.

The isospin averaged $K^{-}$production cross section from nucleon-nucleon collisions is taken from [3] in the parametrization $\left(K=\left(K^{0}, K^{+}\right), \bar{K}=\left(\bar{K}^{0}, K^{-}\right)\right)$

$$
\sigma_{N N \rightarrow N N K \bar{K}}(s) \approx a\left(1-\frac{s_{0}}{s}\right)^{3.17}\left(\frac{s_{0}}{s}\right)^{1.96}
$$

with $a=1.5 \mathrm{mb}$ and $\sqrt{s_{0}}=2 m_{N}+m_{K}^{0}+m_{K}^{*}$. The channels $N \Delta$ and $\Delta \Delta$ are taken to be the same as (16) due to the $K \bar{K}$ pair in the final state. The latter channels play an essential role due to the formation of resonance matter in the heavy-ion collision zone [40] and due to lack of experimental information provide a major source of uncertainty in the present analysis. We will come back to this question in Sec. 3.

Our parametrizations for the $K^{+}$and $K^{-}$cross section from $p p$ collisions are displayed in Fig. 2 by the solid lines in comparison to the inclusive experimental data for $K^{+}$production (open squares) and $K^{-}$production (full circles) as a function of the invariant energy above threshold. The cross section for $K^{+}$production includes both $\Lambda$ and $\Sigma$-hyperon reaction channels. The dash-dotted line (denoted by Z\&S) reflects the parametrization from Ref. [28] that has been used so far [26, 16] for studies of $K^{-}$ production in heavy-ion collisions and close to threshold is much larger than our cross section for $K^{-}$by more than an order of magnitude. Our novel parametrization for the inclusive $K^{-}$cross section is essentially based on the results of the boson-exchange model from [31 for the isospin averaged cross section close to threshold (open square denoted by OBE in Fig. 2). In this OBE-model the different exclusive isospin channels are related to each other via the same Feynman graphs and a comparison to a much larger set of experimental data can be established. As an example for antikaon production we show in the left upper part of Fig. 3 the result of the calculation for the reaction $p p \rightarrow p n K^{+} \bar{K}^{0}$ in comparison to the experimental data from [41]. Also other channels like $p p \rightarrow p \Lambda K^{+}, p p \rightarrow p \Sigma^{0} K^{+}$or $p p \rightarrow K^{0} \Sigma^{+} p$ are described reasonably well within this OBE approach as seen from Fig. 3. We thus expect our new parametrization to 
be more realistic than that of Ref. [28]. However, a detailed experimental study close to threshold energies should be performed to obtain final numbers for the elementary production channels. We finally note that the ratio of the $K^{+} / K^{-}$production cross section increases when going closer to threshold; this is expected because the final phase space for $K^{+}$production is of three-body type while it is a four-body phase space in case of antikaon production.

The experimental $\pi^{-} p \rightarrow p K^{0} K^{-}$cross section, furthermore, can be expressed by [31],

$$
\sigma\left(\pi^{-} p \rightarrow p K^{0} K^{-}\right)=1.121\left(1-\frac{s_{0}}{s}\right)^{1.86}\left(\frac{s_{0}}{s}\right)^{2}[m b],
$$

where $\sqrt{s}$ is the invariant mass of the $\pi N$ system and $\sqrt{s_{0}}=m_{N}+m_{K}^{0}+m_{K}^{*}$. Exploring isospin symmetries 31 the other cross sections can be related to $\sigma\left(\pi^{-} p \rightarrow p K^{0} K^{-}\right)$ by:

$$
\begin{aligned}
2 \sigma\left(\pi^{+} p \rightarrow p K^{+} \bar{K}^{0}\right) & =2 \sigma\left(\pi^{+} n \rightarrow n K^{+} \bar{K}^{0}\right)= & \sigma\left(\pi^{+} n \rightarrow p K^{+} K^{-}\right)= \\
\sigma\left(\pi^{+} n \rightarrow p K^{0} \bar{K}^{0}\right) & =\sigma\left(\pi^{0} p \rightarrow n K^{+} \bar{K}^{0}\right)= & 4 \sigma\left(\pi^{0} p \rightarrow p K^{+} K^{-}\right)= \\
4 \sigma\left(\pi^{0} p \rightarrow p K^{0} \bar{K}^{0}\right) & =\sigma\left(\pi^{0} n \rightarrow p K^{0} K^{-}\right)= & 4 \sigma\left(\pi^{0} n \rightarrow n K^{+} K^{-}\right)= \\
4 \sigma\left(\pi^{0} n \rightarrow n K^{0} \bar{K}^{0}\right) & =2 \sigma\left(\pi^{-} p \rightarrow p K^{0} K^{-}\right)= & \sigma\left(\pi^{-} p \rightarrow n K^{+} K^{-}\right)= \\
\sigma\left(\pi^{-} p \rightarrow n K^{0} \bar{K}^{0}\right) & =2 \sigma\left(\pi^{-} n \rightarrow n K^{0} K^{-}\right) . &
\end{aligned}
$$

These isospin relations have been found in [31] to be well in line with the experimental data from [42.

A further important production channel is given by the flavor exchange reaction $\pi \Lambda \rightarrow K^{-} N$ and $\pi \Sigma \rightarrow K^{-} N$, where the strange quark from the hyperon is exchanged with a light $(\mathrm{u}, \mathrm{d})$ quark. The inverse reaction is the dominant channel for $K^{-}$absorption on nucleons. The latter absorption cross section from Ref. 43 is displayed in Fig. . 1 by the solid line together with the elastic $K^{-} N$ cross section (dashed line) as a function of the $K$-meson momentum with respect to the nucleon at rest. It should be noted that these cross sections are rather well known experimentally [42] and that our parametrization provides an optimal fit through the data.

Apart from the antikaon final state interactions shown in Fig. A, $K^{+}$elastic scattering with nucleons also has an impact on the final kaon spectra. The elastic cross section

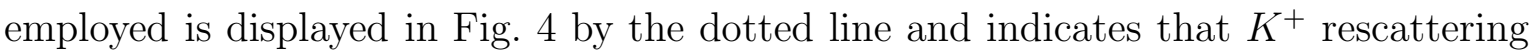
is of minor importance for our present study; however, it is explicitly included in the actual calculations.

By using detailed balance, i.e.

$$
\sigma_{\pi Y \rightarrow \bar{K} N}(s)=\frac{\left[s-\left(m_{K}^{0}+m_{N}\right)^{2}\right]\left[s-\left(m_{K}^{0}-m_{N}\right)^{2}\right]}{\left.\left.\left[s-\left(m_{\pi}+m_{Y}\right)^{2}\right)\right]\left[s-\left(m_{\pi}-m_{Y}\right)^{2}\right)\right]} \sigma_{\bar{K} N \rightarrow \pi Y}(s),
$$

the $\pi$-hyperon production channels can be computed from the parametrizations of the isospin averaged $\bar{K} N \rightarrow \pi Y$ cross sections [44]. The corresponding results are displayed in Fig. 5 as a function of the invariant energy $\sqrt{s}$ (solid and dashed line). Due to the rather well known data for $K^{-} N$ scattering [42] these flavor exchange reactions are expected to be well determined in the actual calculations. 
In addition, we include the $K \bar{K}$ production by $\pi \pi$ reactions; the isospin averaged cross section for this channel can be parametrized by [18]

$$
\bar{\sigma}_{\pi \pi \rightarrow K \bar{K}} \approx a\left(1-\frac{s_{0}}{s}\right)^{0.76}
$$

with $a=2.7 \mathrm{mb}$ and $s_{0}=\left(m_{K}+m_{\bar{K}}\right)^{2}$. This cross section is shown also in Fig. 5 by the dash-dotted line. As we will find in Sect. 3, the contribution of the $\pi \pi$ channel will be less than $5 \%$ up to bombarding energies of $1.85 \mathrm{GeV} / \mathrm{u}$ and could be neglected here.

We, furthermore, have incorporated the antikaon production by hyperon-baryon collisions, which is evaluated in a OBE approach, too. The resulting cross sections are displayed in Fig. 6 by the dashed and solid line which maxima below $1 \mathrm{mb}$. We will find in Sect. 3 that also these channels are of minor importance since their contribution is below $1 \%$ up $1.85 \mathrm{GeV} / \mathrm{u}$.

We note again that the cross sections shown in Figs. 2 6 should account reasonably well for the vacuum scattering processes, but it is not yet clear if a scaling of the cross sections with their threshold values $\sqrt{s_{0}}$, which include the in-medium hadron masses, and the corresponding scaling of the $\Delta N$ cross sections, is the right recipe to adopt. This holds for the antikaon production as well as reabsorption channels and has to be kept in mind when comparing our calculations to experimental data.

\section{$3 \quad \pi^{-}, K^{+}$and $K^{-}$yields for $\mathrm{Ni}+\mathrm{Ni}$ reactions}

The calculation of 'subthreshold' particle production is described in detail in Ref. [1, 45] and has to be treated perturbatively in the energy regime of interest here due to the small cross sections involved. Since we work within the parallel ensemble algorithm, each parallel run of the transport calculation can be considered approximately as an individual reaction event, where binary reactions in the entrance channel at given invariant energy $\sqrt{s}$ lead to final states with 2 (e.g. $K^{+} Y$ in $\pi B$ channels), 3 (e.g. for $K^{+} Y N$ channels in $B B$ collisions) or 4 particles (e.g. $K \bar{K} N N$ in $B B$ collisions) with a relative weight $W_{i}$ for each event $i$ which is defined by the ratio of the production cross section to the total hadron-hadron cross section?. The perturbative treatment now implies that in case of strangeness production channels the initial hadrons are not modified in the respective final channel. On the other hand, each strange hadron is represented by a testparticle with weight $W_{i}$ and propagated according to the Hamilton equations of motion. Elastic and inelastic reactions with pions, $\eta$ 's or nonstrange baryons are computed in the standard way [1, 46] and the final cross section is obtained by multiplying each testparticle with its weight $W_{i}$. In this way one achieves a realistic simulation of the strangeness production, propagation and reabsorption during the heavy-ion collision, where only the dynamical feedback of the strange hadrons to the nonstrange mesons and baryons is neglected.

\footnotetext{
${ }^{3}$ The actual final states are chosen by Monte Carlo according to the 2, 3, or 4-body phase space.
} 
We start our analysis with the system $\mathrm{Ni}+\mathrm{Ni}$ at $1.85 \mathrm{GeV} / \mathrm{u}$ without including any selfenergies for the antikaons. The inclusive Lorentz-invariant cross for negative pions and antikaons in the nucleus-nucleus cms (for $\theta_{l a b}=0^{\circ}$ ) is shown in Fig. 7 by the solid lines in comparison to the data of Ref. [27] that were taken at $0^{\circ}$ in the laboratory system and have been transformed to the nucleus-nucleus cms. We note that our calculations yield an anisotropic $\pi^{-}$angular distribution in this reference frame in line with the analysis in Ref. [47]; however, the $K^{-}$angular distribution is found to be isotropic within the numerical accuracy.

Whereas the $\pi^{-}$spectra in Fig. 7 are reasonably well reproduced - as in many other reactions in this energy regime [46, 48, 47] - the antikaon spectra are underestimated by up to a factor of 6-7. This finding agrees qualitatively with that of Li et al. in Ref. [26], who also underestimated these antikaon data substantially when using a vacuum $K^{-}$ mass, even when adopting the parametrization of the elementary cross section from Ref. 28 (dash-dotted line in Fig. 2).

It is, however, interesting to have a look at the contributions from the different production channels in this case (cf. Fig. 8) in comparison to the experimental data from [27] (full squares) and the preliminary data from the KaoS collaboration [49] for $\mathrm{Ni}+\mathrm{Ni}$ at $1.8 \mathrm{GeV} / \mathrm{u}$. Here the $B B$ channels are approximately in the same order of magnitude as the $\pi B$ channels, but the $\pi Y$ channels provide the dominant contribution as also found in Ref. [16]. This is due to the fact that in more central collisions the pion density reaches about $0.15 \mathrm{fm}^{-3}$ while the hyperons have almost the same abundancy as the $K^{+}$mesons. Thus a substantial amount of hyperons suffer a quark exchange $(s \rightarrow u, d)$ when propagating out of the nuclear medium. We note, furthermore, that for $\mathrm{Ni}+\mathrm{Ni}$ at $1.85 \mathrm{GeV} / \mathrm{u}$ the $\pi \pi$-antikaon production channel contributes by about $3 \%$ whereas the hyperon-nucleon production channels are below $1 \%$.

In order to demonstrate the sensitivity of our results to the $B B$ production cross section employed we show in Fig. 8 by the upper dot-dot-dashed line (denoted by Z\&S) the total $K^{-}$spectrum when adopting the parametrization from Ref. [28]. Here the $B B$ channel becomes the most important one whereas the pion-hyperon channel is about $30 \%$ on average. Even in this limit the experimental spectra are underestimated by about a factor of 4 .

In order to demonstrate more clearly the relevant range of the elementary $K^{-}$cross section above threshold, we display in Fig. 9 the distribution in the baryon-baryon collision number versus invariant energy $\sqrt{s}$, i.e. $d N / d \sqrt{s}$ (histogram), together with the parametrization from Ref. [28] (dot-dashed line) and Eq. (16) (solid line). It is clearly seen that the dominant contributions stem from collisions with invariant energies far below the first experimental data point and that an ambiguity remains as long as our parametrization is not controlled by experimental measurements close to threshold.

Before addressing the in-medium modifications of the antikaons, we show our results for the inclusive $K^{+}$invariant cross section for $\mathrm{Ni}+\mathrm{Ni}$ at $0.8,1.0$ and $1.8 \mathrm{GeV} / \mathrm{u}$ at $\theta_{l a b}=44^{\circ}$ in Fig. 10, that have been transformed to the nucleus-nucleus cms. Also depicted in Fig. 10 are the preliminary $K^{+}$spectra for these systems from Ref. [50]. In comparison we find that for positive kaons apparently no selfenergy effects are needed to describe the data at the energies of interest in line with the expected density 
dependence in Fig. 1. This finding is in accordance with our earlier studies on $K^{+}$ production in nucleus-nucleus [2, 9, 45] and proton-nucleus collisions [51] in line with other groups [52].

We now turn back to the system $\mathrm{Ni}+\mathrm{Ni}$ at $1.85 \mathrm{GeV} / \mathrm{u}$ as well as $1.66 \mathrm{GeV} / \mathrm{u}$ and concentrate on the $K^{-}$spectra at $0^{\circ}$ with respect to the beam axis in the nucleusnucleus cms. In Figs. 11 and 12 we show the effect of antikaon absorption on the spectra in comparison with the data of Ref. [27] and the preliminary data for $\mathrm{Ni}+\mathrm{Ni}$ at $1.8 \mathrm{GeV} / \mathrm{u}$ from Ref. 49. The dashed lines reflect calculations including the bare antikaon mass without any antikaon absorption, while the dash-dotted lines include antikaon absorption as in Fig. 8. We find that $K^{-}$absorption reduces the cross section on average by a factor of 5 for the $\mathrm{Ni}+\mathrm{Ni}$ systems.

We now address the possible medium effects on the antikaon according to Fig. 1 1 or Eq. (9), respectively. We note that for $\alpha=0$ we recover the limit of vanishing antikaon selfenergy, whereas for $\alpha \approx 0.2$ we describe the scenario of Kaplan and Nelson [20] In Figs. 11 and 12 we also display the results of calculations for $\alpha=0.2$ (solid lines). With increasing $\alpha$ not only the magnitude of the spectra is increased, but also the slope becomes softer. For $\alpha \approx 0.2$ (in line with Fig. 1) we still underestimate the experimental spectra slightly. In terms of Eq. (5) this would imply that the 'fundamental' quantity $\Sigma_{K N}$ might be even larger than $350 \mathrm{MeV}$. Anyway, by assuming quite sizeable antikaon attractive selfenergies we can reproduce the data.

\section{Summary}

In this work we have presented a detailed study of kaon and antikaon production in $\mathrm{Ni}+\mathrm{Ni}$ collisions from 0.8 to $1.85 \mathrm{GeV} / \mathrm{u}$ within the coupled channel BUU approach, where the kaons and antikaons are produced perturbatively, however, propagated explicitly with their final state interactions. An important ingredient of our reanalysis of the kaon production cross sections in heavy-ion collisions are the novel elementary production cross sections from Refs. [29, 30, 33, 31] (cf. Sect. 2.2), that are much lower than previous parametrizations used so far in the literature [28, 35]. Furthermore, we have incorporated for the first time all relevant production channels for kaons that are known or can be deduced from hadronic reactions in free space.

Our analysis shows that $\pi^{-}$and $K^{+}$spectra are reasonably well described in this energy regime without introducing any medium modifications for these mesons (cf. Ref. [47] in case of pions). This experience is fully in line with our earlier studies on this subject and the results from independent groups [52, 53. The antikaon spectra, however, are underestimated severely when incorporating only bare kaon masses roughly in line with the study by Li et al. [26]. These authors, however, have used extrapolations for the antikaon cross section, which - to our present knowledge - are

\footnotetext{
${ }^{4}$ For practical purposes one should consider $\alpha$ to be a free parameter to be fixed in comparison to the experimental data in order to learn about the magnitude of the antikaon selfenergy. In fact, we obtain a much better reproduction of the spectra at both bombarding energies for $\alpha \approx 0.24$, but due to the uncertainties involved in the elementary $B B$ production cross sections we cannot determine this value very reliably.
} 
severely overestimated (cf. Fig. 2). When including an attractive antikaon potential comparable to that proposed by Kaplan and Nelson [20], a satisfactory description of the $K^{-}$spectra can be given, both in the actual magnitude as well as in the slope. It is worth noting that the $\pi$-hyperon production channels play a sizeable role in case of the vacuum antikaon mass (Fig. 8), whereas their contribution in the 'dropping mass scenario' of Kaplan and Nelson [20] becomes of minor importance.

The dropping of the antikaon mass with baryon density may be interpreted as a step towards a partial restoration of chiral symmetry that can already be observed at SIS energies. Similar observations have been made at AGS energies [12 as well as at SPS energies, where especially a dropping of the $\rho$-mass can be used to accurately describe the dilepton spectra from heavy-ion reactions [54, 55, 56]. Though there are quite a number of indications for dropping meson masses in the medium by now, one has to properly examine the possibility that conventional many-body effects such as $\Lambda$ hole loops [57] or 'resonance-hole' loops [58 may also account for the spectra observed. These could also affect the selfenergies of hyperons, which are presently treated as 'heavy nucleons'. In addition, we mention again that experimental studies of antikaon production in NN reactions close to threshold are urgently needed to have a final control on the input cross sections used in our present analysis. Another uncertainty in our analysis, i.e. the behaviour of the $\Delta N$ production cross sections, can be eliminated by performing studies for $K^{+}$and $K^{-}$production in proton-nucleus reactions, where the $\Delta$-excitation plays a minor role [59].

The authors acknowledge valuable and inspiring discussions throughout this work with C. M. Ko, P. Senger and Gy. Wolf. 


\section{References}

[1] W. Cassing and U. Mosel, Prog. Part. Nucl. Phys. 25 (1990) 235.

[2] A. Lang, W. Cassing, U. Mosel, and K. Weber, Nucl. Phys. A 541 (1992) 507.

[3] S. Teis, W. Cassing, T. Maruyama, and U. Mosel, Phys. Lett. B 319 (1993) 47; Phys. Rev. C 50 (1994) 388.

[4] C. M. Ko, Q. Li and R. Wang, Phys. Rev. Lett. 59 (1987) 1084; C. M. Ko and Q. Li, Phys. Rev. C 37 (1988) 2270.

[5] X. S. Fang, C. M. Ko, G. Q. Li, and Y. M. Zheng, Phys. Rev. C 49 (1994) R608; Nucl. Phys. A 575 (1994) 766.

[6] G. Q. Li, C. M. Ko, X. S. Fang, and Y. M. Zheng, Phys. Rev. C 49 (1994) 1139; G. Q. Li, C. M. Ko and X. S. Fang, Phys. Lett. B 329 (1994) 149.

[7] K. Weber, B. Blättel, W. Cassing, H.-C. Dönges, V. Koch, A. Lang, and U. Mosel, Nucl. Phys. A 539 (1992) 713.

[8] K. Weber, B. Blättel, W. Cassing, H.-C. Dönges, A. Lang, T. Maruyama, and U. Mosel, Nucl. Phys. A 552 (1993) 571.

[9] T. Maruyama, W. Cassing, U. Mosel, S. Teis, and K. Weber, Nucl. Phys. A 573 (1994) 653.

[10] J. Aichelin, Phys. Reports 202 (1991) 233; J. Aichelin and H. Stöcker, Phys. Lett. B 176 (1986) 14; J. Aichelin, G. Peilert, A. Bohnet, A. Rosenhauer, H. Stöcker, and W. Greiner, Phys. Rev. C 37 (1988) 2451.

[11] H. Sorge, H. Stöker, and W. Greiner, Ann. Phys. 192 (1989) 266; Nucl. Phys. A 498 (1989) 567c; H. Sorge, A. V. Keitz, R. Matiello, H. Stöcker, and W. Greiner, Z. Phys. C 47 (1990) 629; Phys. Lett. B 243 (1990) 7; A. Jahns, H. Sorge, H. Stöcker, and W. Greiner, Z. Phys. A 341 (1992) 243.

[12] W. Ehehalt and W. Cassing, Nucl. Phys. A 602 (1996) 449.

[13] W. Cassing, A. Lang, S. Teis, and K. Weber, Nucl. Phys. A 545 (1992) 123c.

[14] G. Batko, A. Faessler, S.W. Huang, E. Lehmann, and R.K. Puri, J. Phys. G 20 (1994) 461.

[15] C. M. Ko, Phys. Lett. B 120 (1983) 294; ibid. B 138 (1984) 361.

[16] H. W. Barz and H. Iwe, Phys. Lett. B 153 (1985) 217.

[17] S. W. Huang, G. Q. Li, T. Maruyama, and A. Faessler, Nucl. Phys. A 547 (1992) 653. 
[18] G. E. Brown, C. M. Ko, Z. G. Wu, and L. H. Xia, Phys. Rev. C 43 (1991) 1881.

[19] G. E. Brown and M. Rho, Phys. Rev. Lett. 66 (1991) 2720.

[20] D. B. Kaplan and A. E. Nelson, Phys. Lett. B 175 (1986) 57.

[21] G. E. Brown, K. Kubodera, and M. Rho, Phys. Lett. B 192 (1987) 273; G. E. Brown, C. M. Ko, and K. Kubodera, Z. Phys. A 341 (1992) 301.

[22] C. H. Lee, G. E. Brown, D. P. Min, and M. Rho, Nucl. Phys. A 585 (1995) 401.

[23] G. Q. Li and C. M. Ko, Phys. Lett. B 338 (1994) 118.

[24] M. Rho, 'International Nuclear Physics Conference', Aug. 1995, Beyjing, China, to appear in Nucl. Phys. A.

[25] M. Lutz, A. Stein and W. Weise, Nucl. Phys. A 574 (1994) 755.

[26] G. Q. Li, C. M. Ko, and X. S. Fang, Phys. Lett. B 329 (1994) 149.

[27] A. Schröter et al., Z. Phys. A350 (1994) 101.

[28] W. Zwermann and B. Schürmann, Phys. Lett. B145 (1984) 315.

[29] A. Sibirtsev et al., Z. Phys. A 351 (1995) 333.

[30] A. Sibirtsev, preprint UGI 96-20.

[31] A. Sibirtsev, W. Cassing and C. M. Ko, preprint UGI 96-21.

[32] G. I. Lykasov, M. V. Rzjanin and W. Cassing, nucl-th/9609018, Phys. Lett. B, in print.

[33] A. Sibirtsev, W. Cassing, and U. Mosel, nucl-th/9607047.

[34] J. Q. Wu and C. M. Ko, Nucl. Phys. A 499 (1989) 810; G. Q. Li and C. M. Ko, Nucl. Phys. A 594 (1995) 439.

[35] J. Randrup and C. M. Ko, Nucl. Phys. A 343 (1980) 519; A 411 (1983) 537.

[36] B. Schürmann and W. Zwermann, Phys. Lett. B 183 (1987) 31.

[37] A. E. Nelson and D. Kaplan, Phys. Lett. B 192 (1987) 193.

[38] G. Q. Li and C.M. Ko, Nucl. Phys. A 582 (1995) 731.

[39] K. Tsushima, S. W. Huang, and A. Faessler, J. Phys. G 21 (1995) 33; Phys. Lett B 337 (1994) 245.

[40] W. Ehehalt, W. Cassing, A. Engel, U. Mosel, and Gy. Wolf, Phys. Rev. C 47 (1993) R2467. 
[41] G. Giacomelli, Int. J. Mod. Phys. A 5 (1990) 223.

[42] Landolt-Börnstein, New Series, ed. H. Schopper, I/12 (1988).

[43] S. V. Efremov and E. A. Paryev, Z. Phys. A 348 (1994) 217.

[44] J. Cugnon, P. Deneye and J. Vandermeulen, Phys. Rev. C 41 (1990) 1701.

[45] W. Cassing, V. Metag, U. Mosel, and K. Niita, Phys. Rep. 188 (1990) 363.

[46] Gy. Wolf, W. Cassing, W. Ehehalt, and U. Mosel, Prog. Part. Nucl. Phys. 30 (1993) 273.

[47] S. Teis, W. Cassing, M. Effenberger, A. Hombach, U. Mosel, and Gy. Wolf, hepth/9609009, Z. Phys. A, in print.

[48] W. Ehehalt, W. Cassing, A. Engel, U. Mosel, and Gy. Wolf, Phys. Lett. B 298 (1993) 31.

[49] P. Senger, to appear in APH N.S., Heavy Ion Physics, (1996).

[50] R. Barth et al., GSI Annual Report 1995, p. 51.

[51] W. Cassing, G. Batko, U. Mosel, K. Niita, O. Schult, and Gy. Wolf, Phys. Lett. B 238 (1990) 25.

[52] C. Hartnack, J. Jaenicke, L. Sehn, H. Stoecker, and J. Aichelin, Nucl. Phys. A 580 (1994) 643.

[53] S. A. Bass, C. Hartnack, H. Stoecker, W. Greiner, Phys. Rev. C 51 (1995) 12.

[54] W. Cassing, W. Ehehalt, and C. M. Ko, Phys. Lett. B 363 (1995) 35.

[55] G. Q. Li, C. M. Ko and G. E. Brown, Phys. Rev. Lett. 75 (1995) 4007.

[56] W. Cassing, W. Ehehalt, and I. Kralik, Phys. Lett. B 377 (1996) 5.

[57] T. Wass, N. Kaiser, and W. Weise, Phys. Lett. B 365 (1996) 12; B 379 (1996) 34.

[58] B. Friman, QUARK MATTER '96, Heidelberg, May 1996, ed. P. Braun-Munzinger et al., to appear in Nucl. Phys. A.

[59] W. Cassing, T. Demski, L. Jarczyk, B. Kamys, Z. Rudy, O. W. B. Schult, and A Strzalkowski, Z. Physik A 349 (1994) 77. 


\section{Figure Captions}

Figure 1: The $K^{+}$and $K^{-}$mass as a function of the baryon density in units of $\rho_{0} \approx 0.16 \mathrm{fm}^{-3}$ according to Kaplan and Nelson (Eq. (5)). The dashed line (with $\alpha=$ $0.2)$ presents a linear fit to the $K^{-}$effective mass.

Figure 2: The parametrizations for the isospin averaged inclusive $K^{+}$and $K^{-}$cross sections from $N N$ collisions as a function of the invariant energy $\sqrt{s}$ above threshold $\sqrt{s_{0}}$ (solid lines) in comparison to the experimental data [41]. The dash-dotted line is the parametrization from Ref. 28] for $K^{-}$production whereas the open square (denoted by OBE) is from the OBE calculation of Ref. [31].

Figure 3: Comparison of our parametrizations with the experimental cross sections 42 for the isospin channels $p p \rightarrow p n K^{+} \bar{K}^{0}, p p \rightarrow p \Lambda K^{+}, p p \rightarrow p \Sigma^{0} K^{+}, p p \rightarrow p \Sigma^{+} K^{0}$.

Figure 4: $K^{-} N$ inelastic (solid line) and elastic (dashed line) cross section as a function of kaon laboratory momentum $p_{K}$ as fitted to the experimental data from [42]. The dotted line displays the elastic $K^{+} N$ cross section.

Figure 5: Antikaon production cross section from $\pi+$ hyperon collisions according to Eq. (12). The dash-dotted line represents the channel $\pi \pi \rightarrow K \bar{K}$ according to Eq. (20).

Figure 6: Antikaon production cross sections from hyperon-nucleon collisions according to an OBE calculation.

Figure 7: The inclusive Lorentz-invariant cross section as a function of the meson momentum in the nucleus-nucleus cms for $\pi^{-}$and $K^{-}$mesons at $\theta_{\text {lab }}=0^{\circ}$ for $\mathrm{Ni}+\mathrm{Ni}$ at $1.85 \mathrm{GeV} / \mathrm{u}$. The full dots and full squares represent the experimental data from Ref. [27. In these calculations no meson selfenergies have been taken into account. 
Figure 8: The inclusive Lorentz-invariant cross section as a function of the kaon momentum in the nucleus-nucleus cms for $K^{-}$mesons at $\theta_{l a b}=0^{\circ}$ for $\mathrm{Ni}+\mathrm{Ni}$ at 1.85 $\mathrm{GeV} / \mathrm{u}$ without including $K^{-}$selfenergies in comparison to the experimental data from [27] and the preliminary data for $\mathrm{Ni}+\mathrm{Ni}$ at $1.8 \mathrm{GeV} / \mathrm{u}$ from [49]. The dashed line displays the cross section from baryon-baryon $(B B)$ channels, the dotted line that from pion-baryon $(\pi B)$ channels, while the dash-dotted line shows the contribution from $\pi$ hyperon collisions. The solid line represents the sum of all channels taken into account. In addition we show the full result when using the parametrization from Ref. 28] for the $B B$ channel.

Figure 9: The distribution in the $B B$ collision number versus the invariant energy $d N / d s^{1 / 2}$ for $\mathrm{Ni}+\mathrm{Ni}$ at $1.85 \mathrm{GeV} / \mathrm{u}$ (histogram). For orientation we display again our parametrization for the isospin averaged inclusive $K^{-}$cross section (solid line) in comparison to the experimental data and the parametrization from Ref. [28] (dotdashed line).

Figure 10: The inclusive Lorentz-invariant cross section as a function of the meson momentum in the nucleus-nucleus cms for $K^{+}$mesons at $\theta_{\text {lab }}=44^{\circ}$ for $\mathrm{Ni}+\mathrm{Ni}$ at $0.8,1.0$ and $1.8 \mathrm{GeV} / \mathrm{u}$ without including any meson selfenergies. The experimental preliminary $K^{+}$spectra have been taken from Ref. [50].

Figure 11: The inclusive Lorentz-invariant cross section as a function of the kaon momentum in the nucleus-nucleus cms for $K^{-}$mesons at $\theta_{l a b}=0^{\circ}$ for $\mathrm{Ni}+\mathrm{Ni}$ at 1.85 $\mathrm{GeV} / \mathrm{u}$ in comparison to the experimental data from Ref. 27] and the preliminary data for $\mathrm{Ni}+\mathrm{Ni}$ at $1.8 \mathrm{GeV} / \mathrm{u}$ from Ref. 49 . The dashed line corresponds to a calculation with bare kaon masses when discarding $K^{-}$reabsorption, whereas the dash-dotted line includes $K^{-}$reabsorption. The solid line displays the results with antikaon selfenergies Eq. (9) for $\alpha=0.2$ including $K^{-}$reabsorption.

Figure 12: The inclusive Lorentz-invariant cross section as a function of the kaon momentum in the nucleus-nucleus cms for $K^{-}$mesons at $\theta_{l a b}=0^{\circ}$ for $\mathrm{Ni}+\mathrm{Ni}$ at $1.66 \mathrm{GeV} / \mathrm{u}$ in comparison to the experimental data from Ref. [27]. The dashed line corresponds to a calculation with bare kaon masses when discarding $K^{-}$reabsorption, whereas the dash-dotted line includes $K^{-}$reabsorption. The solid line displays the results with antikaon selfenergies Eq. (9) for $\alpha=0.2$ including $K^{-}$reabsorption. 


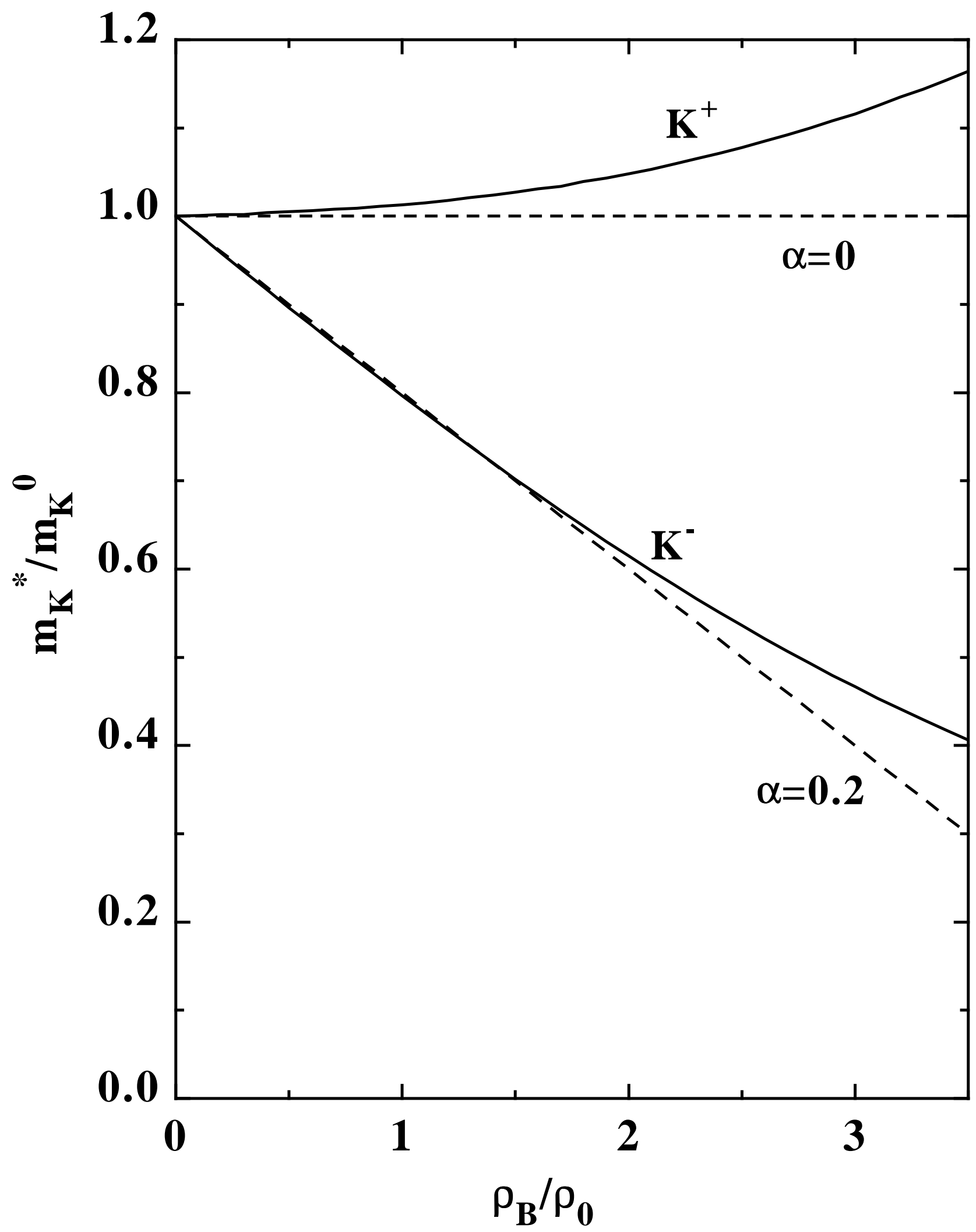

Fig. 1 


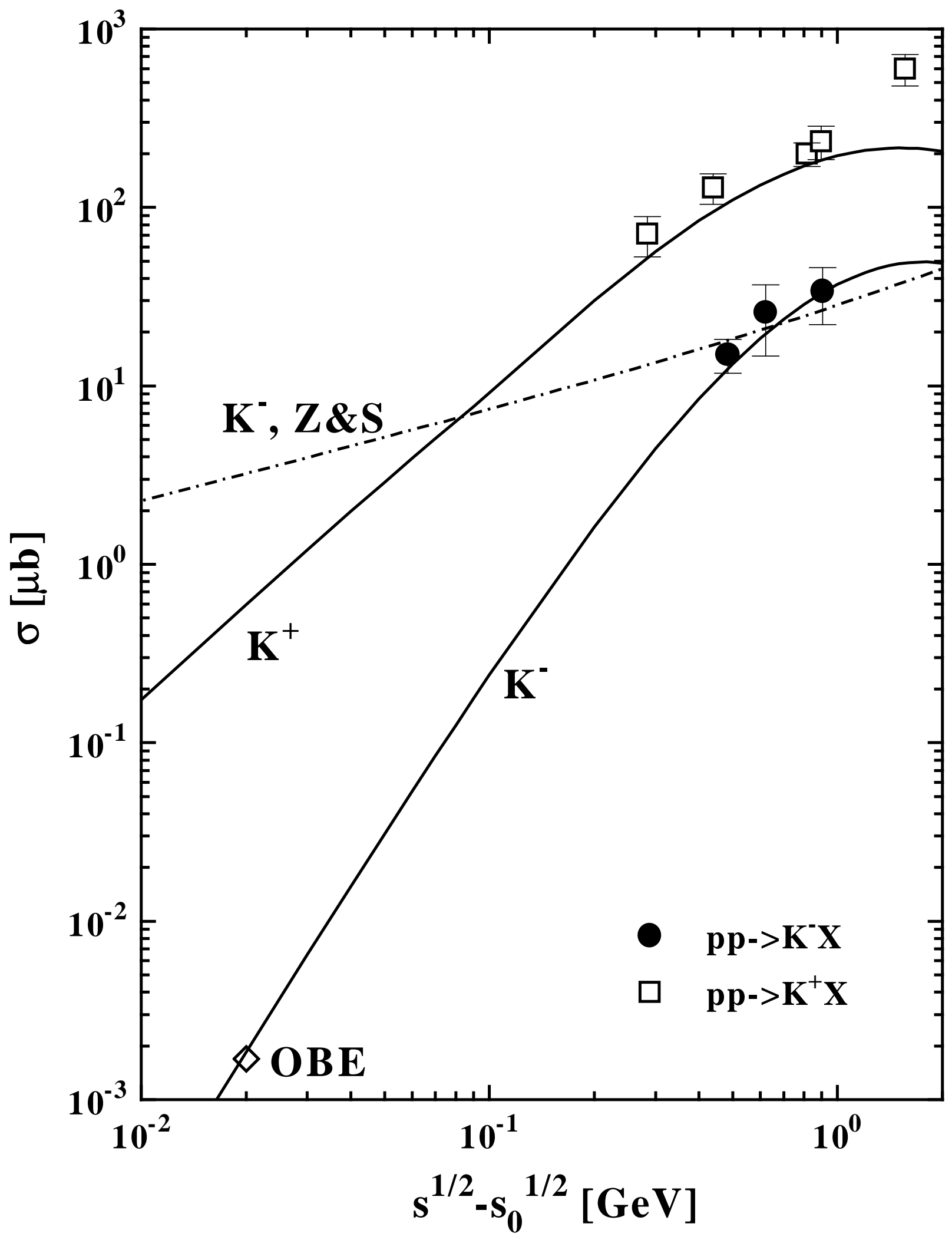

Fig. 2 

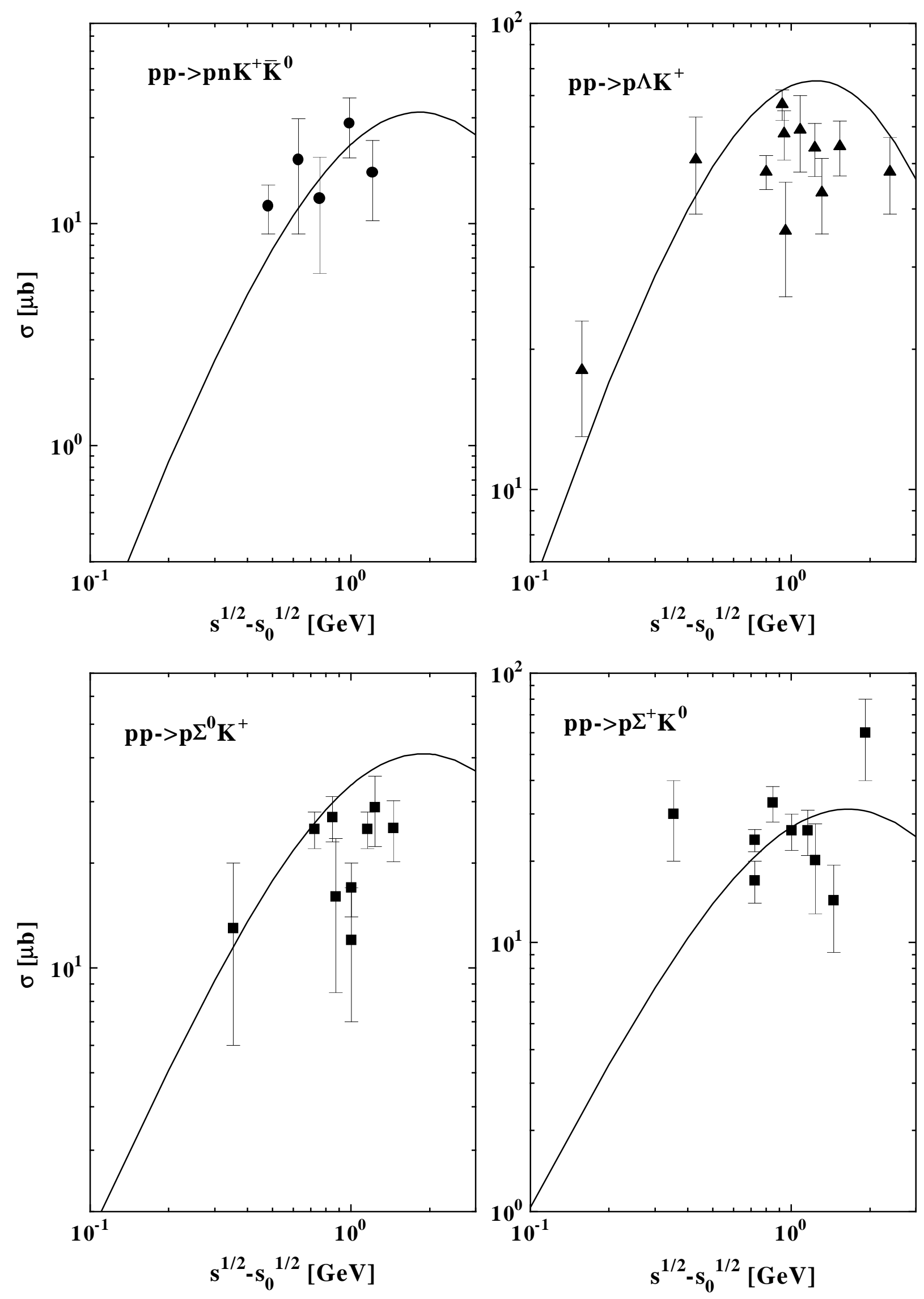

Fig. 3 


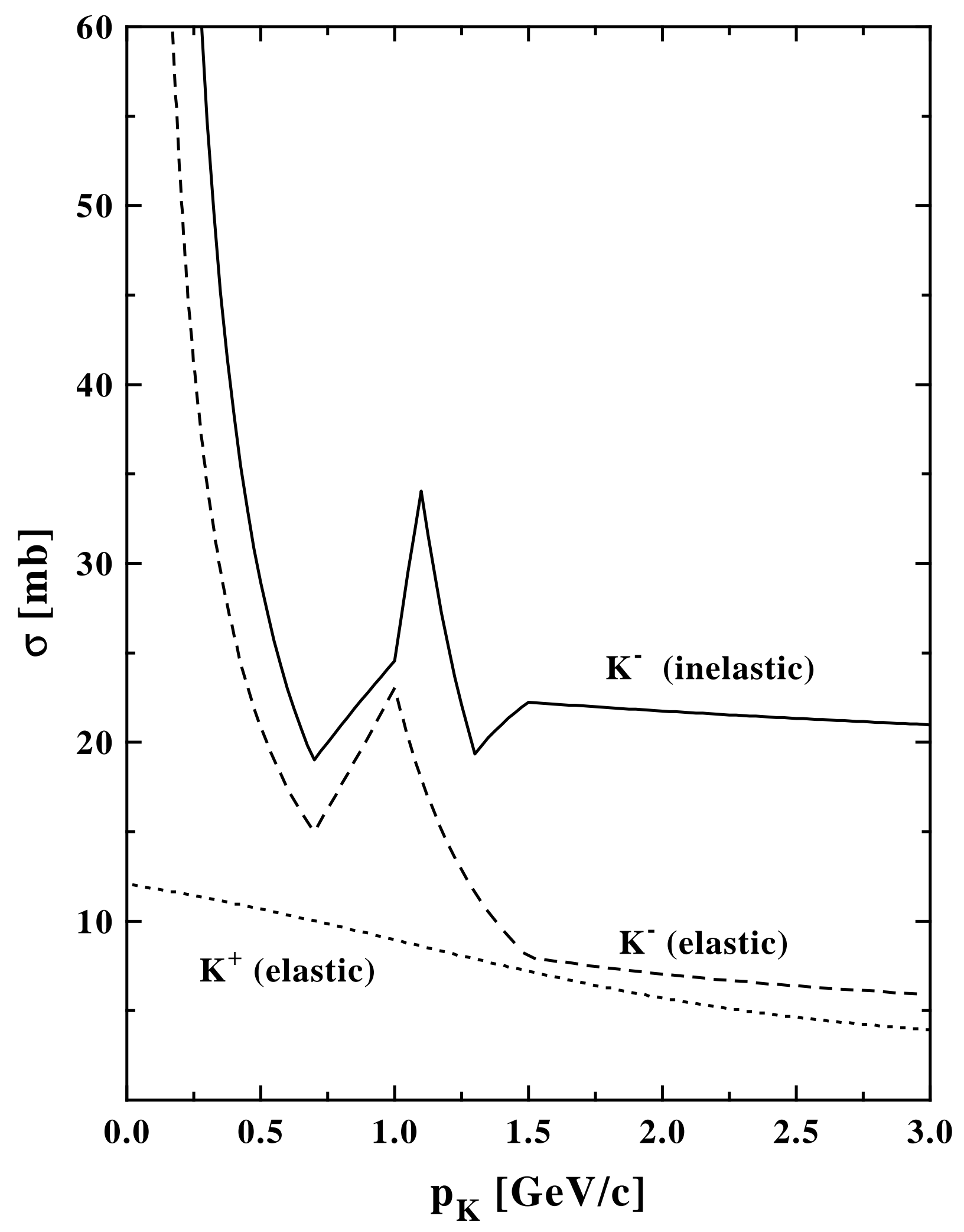

Fig. 4 


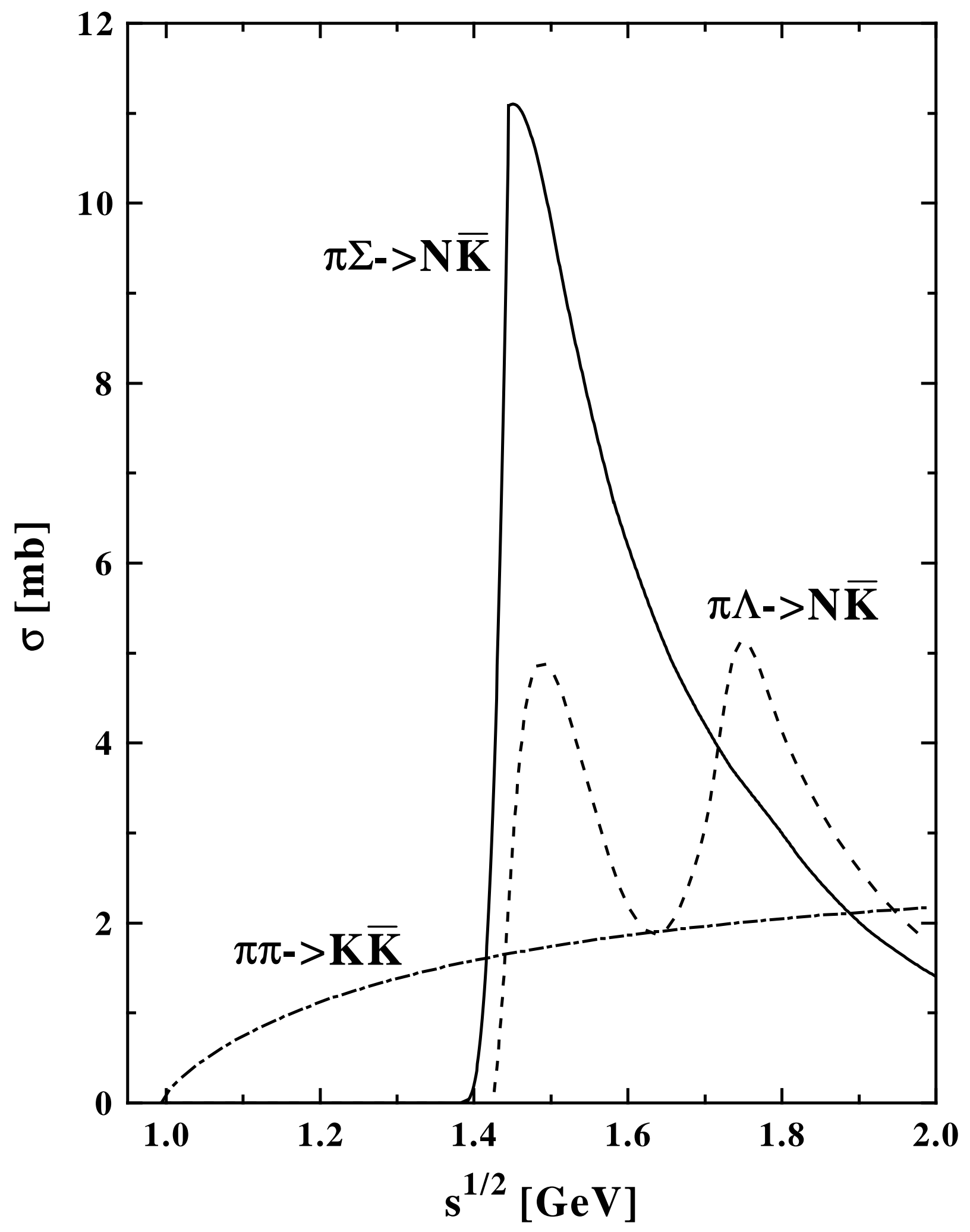

Fig. 5 


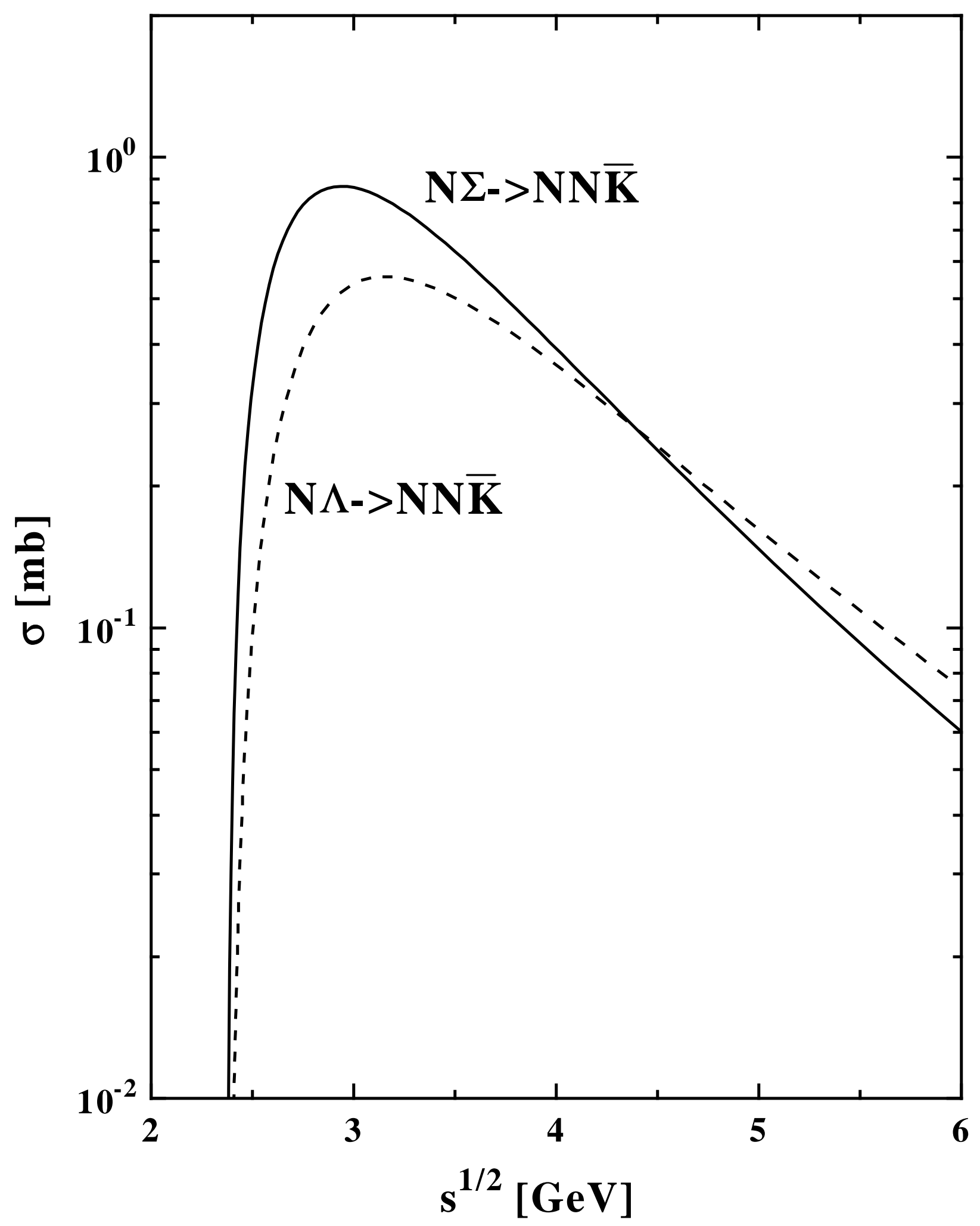

Fig. 6 


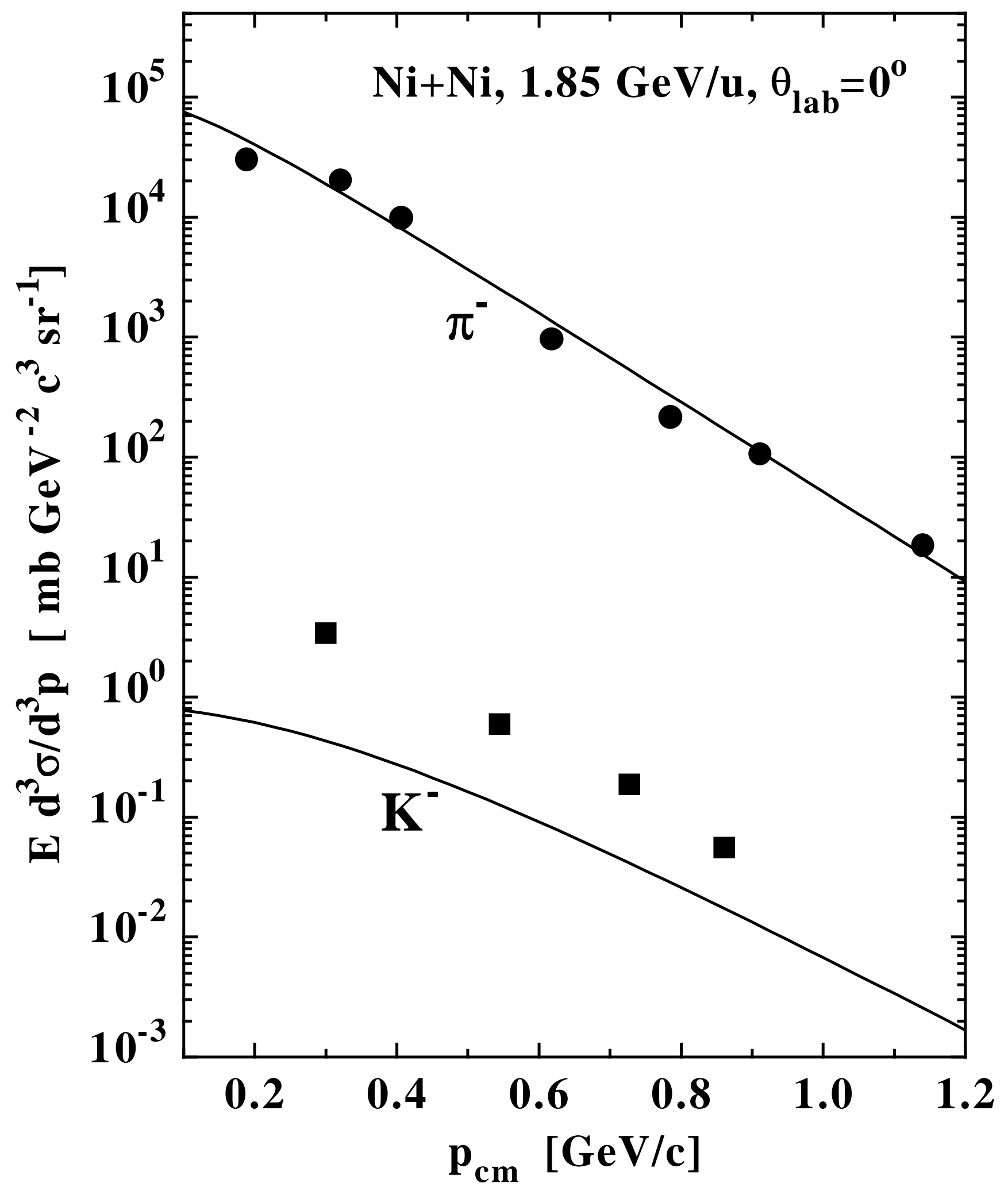

Fig. 7 


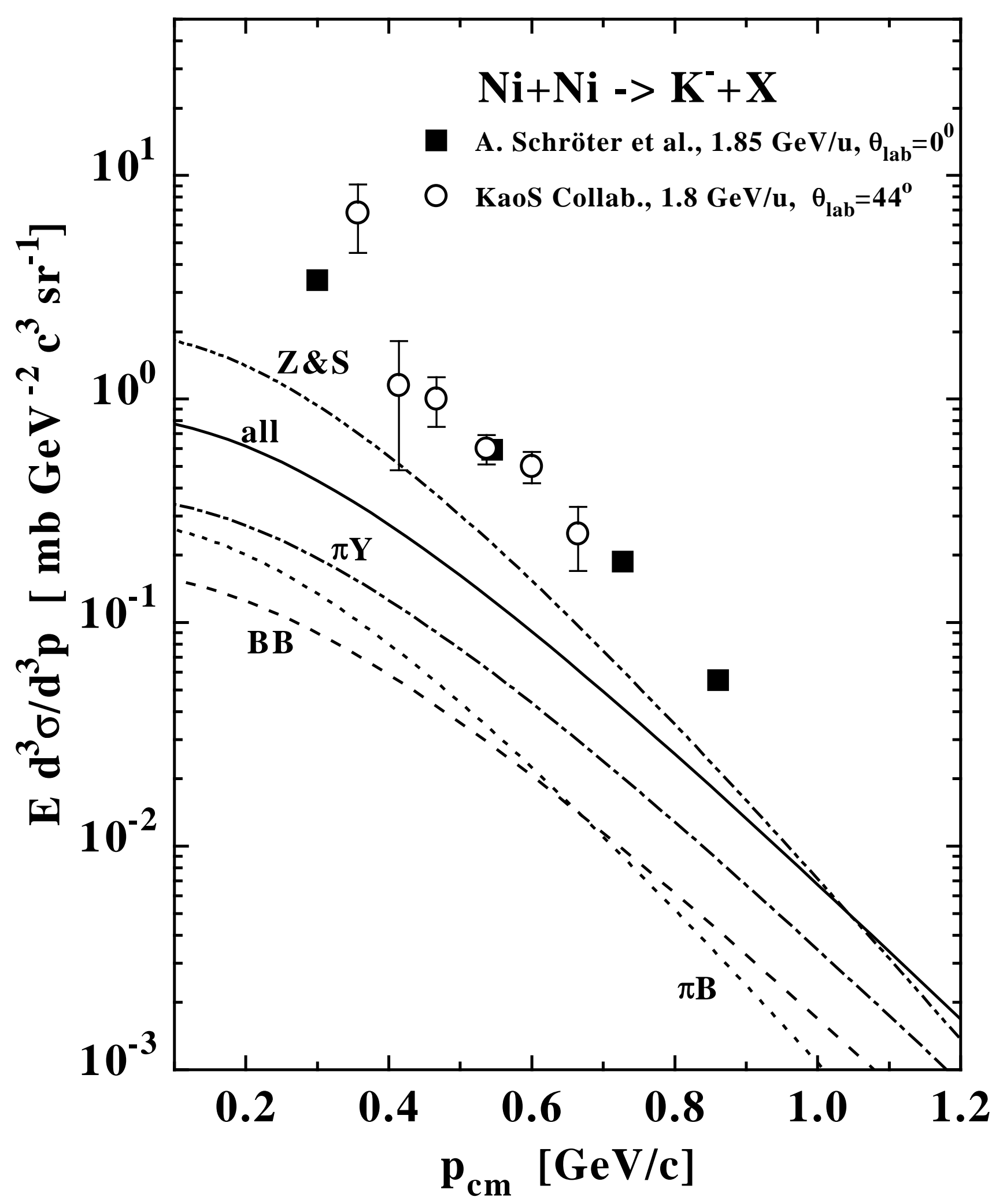

Fig. 8 


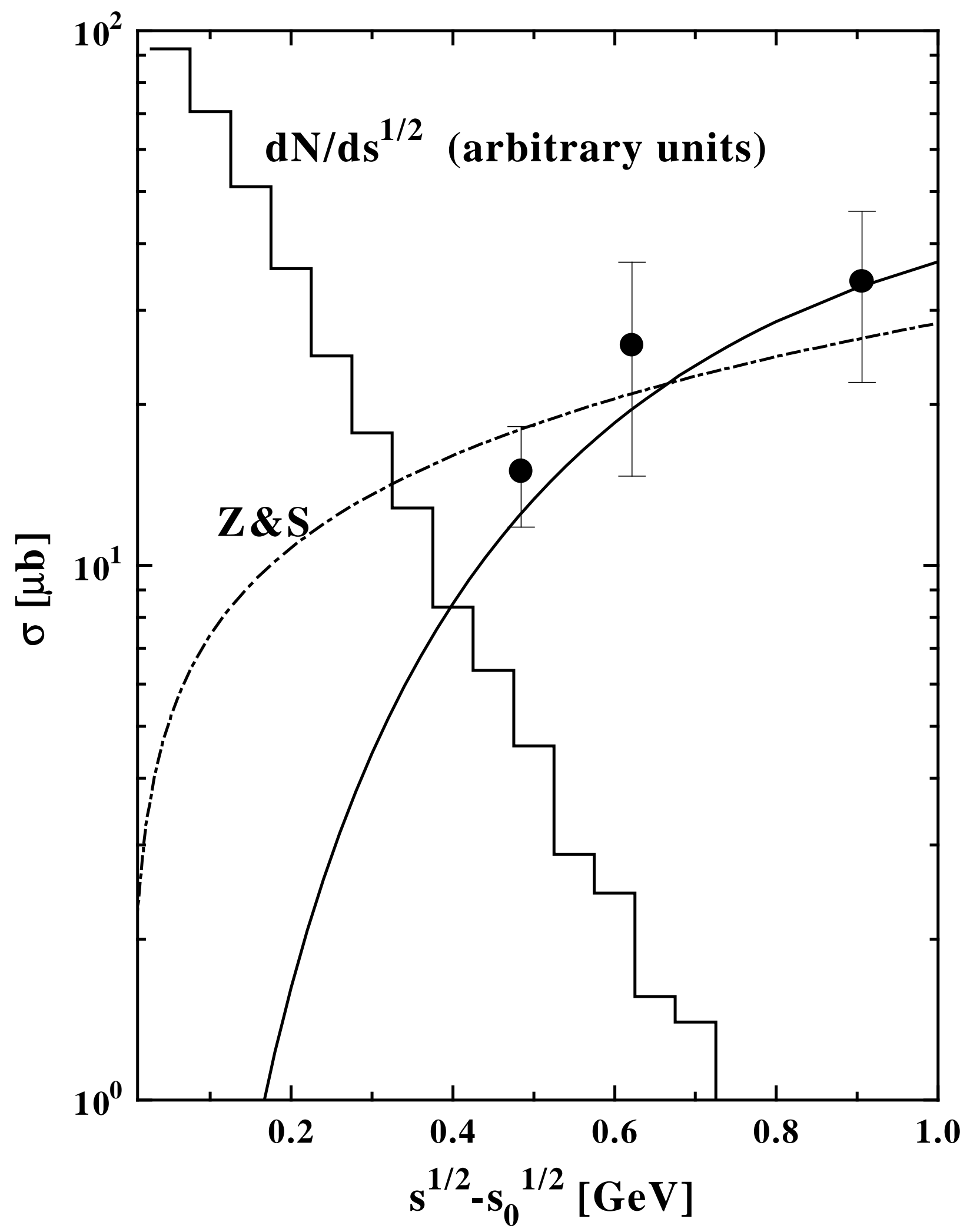

Fig. 9 


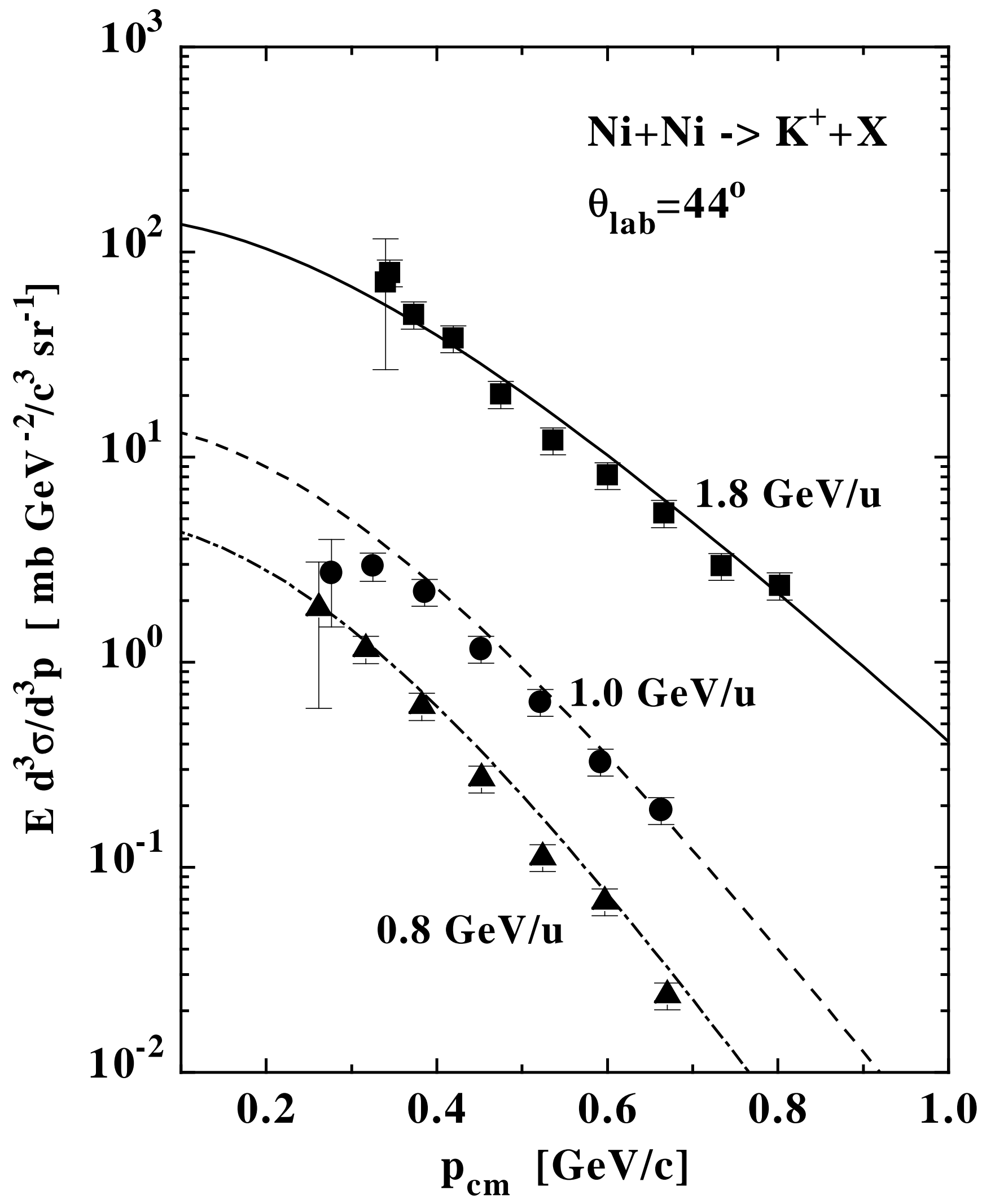

Fig. 10 


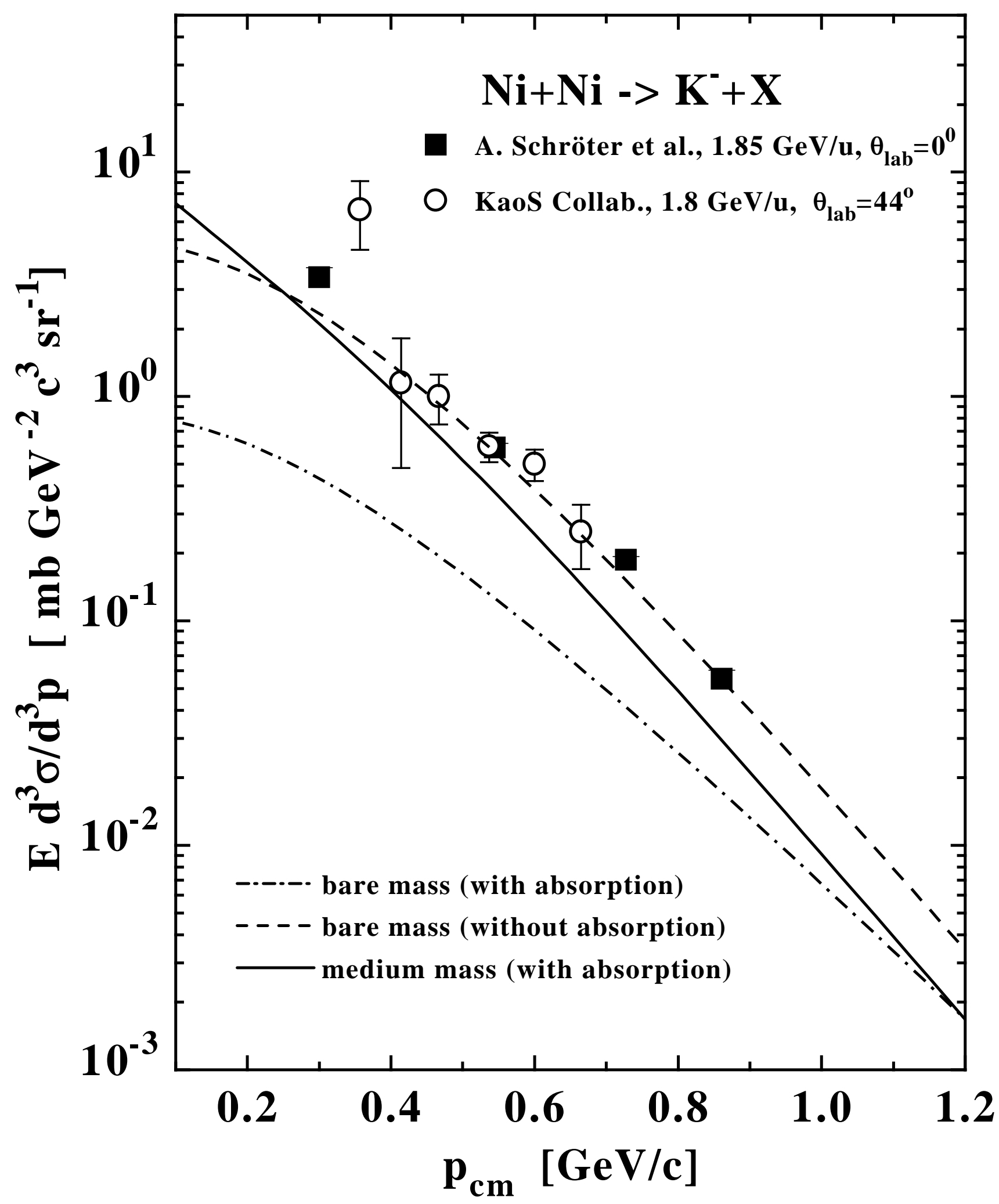

Fig. 11 


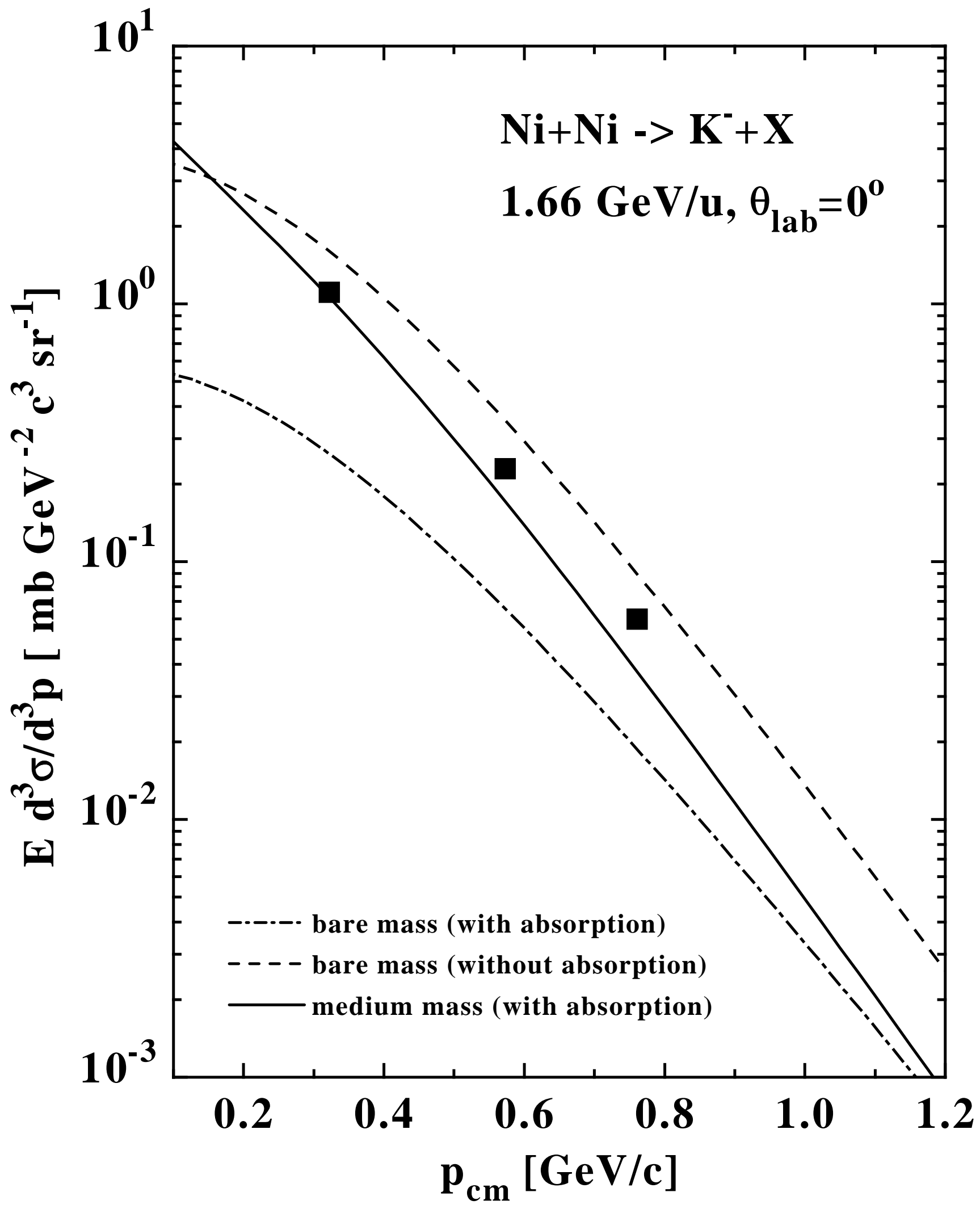

Fig. 12 